\title{
Theoretical Study of Diode Pumped Intracavity Doubled Passively Q-Switched Solid-State Lasers
}

\author{
M. Alshikh Khalil* and B. Abbas \\ Atomic Energy Commission, P.O. Box 6091, Damascus, Syria \\ (Received November 16, 2007; revised version January 14, 2008) \\ In this paper, some designed curves such as peak power, energy, pulse \\ width, and a factor describing the pulse symmetry are generated. With these \\ curves and expressions, one can design a passively Q-switched intracavity \\ frequency-doubled laser and predict the pulse characteristics.
}

PACS numbers: 42.65.Ky, 42.55.Rz, 42.60.Gd, 02.60.Cb

\section{Introduction}

In recent years, great attention has been paid to high peak power all-solid-state pulsed blue-green lasers [1-4] for some applications such as spectroscopy, display, optical data storage, coherent telecommunications, remote sensing, medicine, etc. Such lasers are generally realized by intracavity or external cavity frequency doubling of cw laser-diode (LD)-pumped solid-state Q-switched Nd-doped lasers. The higher nonlinear conversion efficiency from an intracavity doubling laser can be obtained due to its high fundamental wave power density in the laser resonator, which is especially so for a laser with middle or low output power. Actively Q-switched (acousto- or electro-optic switch) laser requires fast driving electronics, resulting in costly and complicated laser systems. In comparison with actively Q-switched lasers, the passively Q-switched ones contain fewer components, and are less expensive, more compact, and more reliable.

Rate equations are efficient tools for analyzing the performance of a Q-switched laser. An accurate and general theoretical model of a LD-pumped passively Q-switched intracavity frequency doubling laser has been studied [5], in which the intracavity photon density is assumed to be Gaussian spatial distributions and the longitudinal variation of the intracavity photon density is also considered. Recently, Brenier et al. [6] verified the last model and report the

*corresponding author; e-mail: scientific@aec.org.sy 
passive Q-switching of a diode pumped Yb:GdA1 ${ }_{3}\left(\mathrm{BO}_{3}\right)_{4}$ (Yb:GAB) laser. They obtained repetition rates in the $1-4.5 \mathrm{kHz}$ range and pulse energies of 125 and $165 \mu \mathrm{J}$ with about $30 \mathrm{~ns}$ duration.

In this paper, we study the effect of the output mirror reflectivity and the initial absorption through the parameter $p$ on the pulse width, the energy, the power, and the symmetry of the output pulse.

\section{Laser rate equations model}

Figure 1 shows the scheme of the diode end-pumped passively Q-switched laser. When the rate equations are used to describe a Q-switched intracavity-frequency-doubling laser, the second harmonic conversion can be usually considered as a nonlinear loss of the fundamental wave [7]. According to frequency-

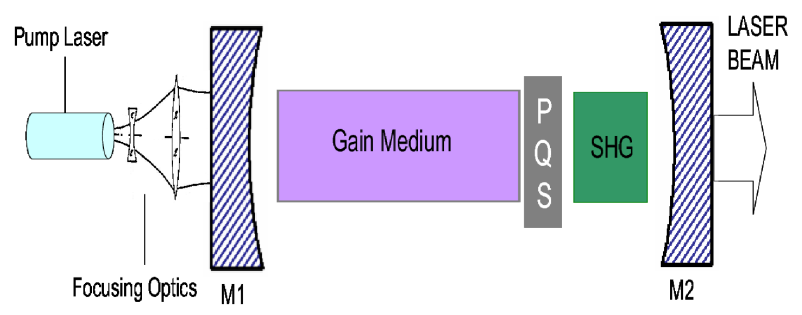

Fig. 1. The experimental setup.

-doubling theory and rate equation theory, assuming the distribution in the transverse section of pump light, and the laser intensities in the gain medium and in the Q-switch absorber uniform, neglecting the influence of longitudinal attenuation of the pump light, we can obtain the coupling rate equations for passively Q-switched intracavity doubling laser with $\mathrm{Cr}^{4+}$ :YAG saturable absorber [7]:

$$
\begin{aligned}
\frac{\mathrm{d} \phi}{\mathrm{d} t} & =\frac{\phi}{t_{\mathrm{r}}}\left[2 \sigma n l-2 \sigma_{13} n_{\mathrm{s} 1} l_{\mathrm{s}}-2 \sigma_{24}\left(n_{\mathrm{s} 0}-n_{\mathrm{s} 1}\right) l_{\mathrm{s}}-(L-\ln R)\right] \\
& -\frac{\eta_{\mathrm{SHG}}}{t_{\mathrm{r}}} \phi^{2} \\
\frac{\mathrm{d} n}{\mathrm{~d} t} & =W_{\mathrm{p}}-\frac{n}{\tau_{\mathrm{g}}}-\gamma \sigma c n \phi, \\
\frac{\mathrm{d} n_{\mathrm{s} 1}}{\mathrm{~d} t} & =\frac{n_{\mathrm{s} 0}-n_{\mathrm{s} 1}}{\tau_{\mathrm{s}}}-\gamma_{\mathrm{s}} \sigma_{13} c \phi n_{\mathrm{s} 1} \frac{A_{\mathrm{g}}}{A_{\mathrm{s}}}
\end{aligned}
$$

where $\phi$ is the fundamental photon density, $t_{\mathrm{r}}=2 l^{\prime} / c$ is the photon round-trip transit time in the laser cavity, $c$ is the speed of light in vacuum, $l^{\prime}$ is the optical cavity length, $l^{\prime}=\left[n_{1} l+n_{2} l_{\mathrm{s}}+n_{3} l_{\mathrm{k}}+\left(L_{\mathrm{c}}-l-l_{\mathrm{s}}-l_{\mathrm{k}}\right)\right], n_{1}$ is the refractive index of the laser crystal, $n_{2}$ is the refractive index of saturable absorber (SA), $n_{3}$ is the refractive index of the nonlinear crystal, $l$ is the length of the gain medium, 
$l_{\mathrm{S}}$ is the thickness of SA, $l_{\mathrm{k}}$ is the length of the nonlinear crystal, $L_{\mathrm{c}}$ is the physical cavity length; $n$ is the instantaneous population inversion density of the laser medium, $n_{\mathrm{s} 1}$ and $n_{\mathrm{s} 0}$ are the instantaneous population density of the absorber in ground state level and the total density of the absorber, respectively; $\eta_{\mathrm{SHG}}$ is a coefficient of the intracavity frequency doubling, $\eta_{\mathrm{SHG}}=K c \hbar \omega l_{\mathrm{k}}^{2}$, $\hbar \omega$ is the single photon energy of the fundamental wave, $\hbar$ is the Planck constant, $\omega$ is the angular frequency of the fundamental beam, $K=2 \omega^{2} d_{\mathrm{eff}}^{2} / c^{3} \varepsilon_{0} n_{\omega}^{2} n_{2 \omega}, d_{\mathrm{eff}}$ (in $[\mathrm{m} / \mathrm{V}]$ ) is the effective nonlinear coefficient of the nonlinear crystal, $\varepsilon_{0}$ is the permittivity of free space, $n_{\omega}, n_{2 \omega}$ are the indices of refraction for the fundamental wave and the second harmonic wave, respectively; $\sigma$ is the laser stimulated emission cross-section, $\sigma_{13}$ and $\sigma_{24}$ are the ground- and excited-state absorption cross-sections of the absorber; $W_{\mathrm{p}}$ is the pumping rate (the number of excited atoms per volume unit per second); $\tau_{\mathrm{g}}$ and $\tau_{\mathrm{s}}$ are the relaxation time for the inverted population difference of the laser and absorber media; $A_{\mathrm{g}}$ and $A_{\mathrm{s}}$ are the effective areas of resonator mode in gain medium and saturable absorber; $\gamma$ and $\gamma_{\mathrm{s}}$ correspond to the net reduction in the population inversion, upon the stimulated emission of a single photon; $R$ is the reflectivity of the output mirror, and $L$ is the residual optical loss including the intrinsic loss of the cavity (the diffraction loss resulting from thermal effect and the insertion loss of the saturable absorber). The four-level model of $\mathrm{Cr}^{4+}$-doped saturable absorbers is shown in Fig. 2 [8].

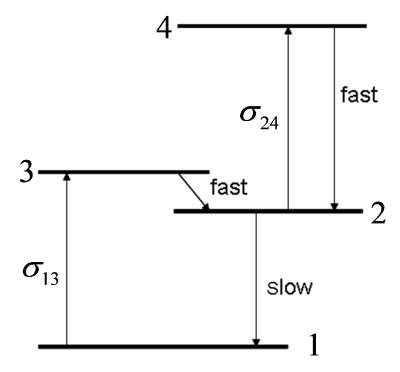

Fig. 2. The four-level model of $\mathrm{Cr}^{4+}$-doped saturable absorber.

The above equations can be solved numerically. Alternatively, we can obtain an analytical form according to the laser dynamics that separates the Q-switching process in two steps. The influence of the optical pumping $W_{\mathrm{p}}$ and the gain relaxation term $-n / \tau_{\mathrm{g}}$ in Eq. (2.1b) as well as the influence of the saturable absorber relaxation term $\left(n_{\mathrm{s} 0}-n_{\mathrm{s} 1}\right) / \tau_{\mathrm{s}}$ in Eq. (2.1c) are neglected. The dynamics is characterized by the rapid growth and decay of the pulse and by the decrease in the gain inversion and of the SA ground state population density. Then, the pulse energy $E_{\mathrm{p}}$ and the peak power $P_{\mathrm{p}}$ could be obtained. Solving the rate equations in the manner Liu and Kim did [7], the equation of the photon density $\phi$ as a function of population inversion $n$ resulted as 


$$
\begin{aligned}
\phi(n) & =\frac{l}{\gamma l^{\prime}} n_{\mathrm{i}}\left\{\frac{1}{1-k}\left[\left(\frac{n}{n_{\mathrm{i}}}\right)^{k}-\left(\frac{n}{n_{\mathrm{i}}}\right)\right]\right. \\
& +\frac{1}{(\alpha-k)} \frac{(1-\delta) \ln T_{0}^{2}}{-\ln R+L-\ln T_{0}^{2}}\left[\left(\frac{n}{n_{\mathrm{i}}}\right)^{k}-\left(\frac{n}{n_{\mathrm{i}}}\right)^{\alpha}\right] \\
& \left.-\frac{1}{k}\left[1+\frac{(1-\delta) \ln T_{0}^{2}}{-\ln R+L-\ln T_{0}^{2}}\right]\left[1-\left(\frac{n}{n_{\mathrm{i}}}\right)^{k}\right]\right\} .
\end{aligned}
$$

Here $\delta=\sigma_{24} / \sigma_{13}$ is a constant which accounts for the excited state absorption relative to the ground state absorption of the $\mathrm{Cr}^{4+}$ :YAG, $T_{0}=\exp \left(-\sigma_{13} n_{\mathrm{s} 0} l_{\mathrm{s}}\right)$ is the initial transmission of the $\mathrm{SA}, n_{\mathrm{i}}=\left(-\ln R+L-\ln T_{0}^{2}\right) / 2 \sigma l$ and $\alpha$ is a parameter determined by the properties of the gain and SA media and on the ratio $A_{\mathrm{g}} / A_{\mathrm{s}}$ :

$$
\alpha=\frac{\gamma_{\mathrm{s}} \sigma_{13}}{\gamma \sigma} \frac{A_{\mathrm{g}}}{A_{\mathrm{s}}}
$$

and

$$
k=\frac{\eta_{\mathrm{SHG}}}{2 \sigma \gamma l^{\prime}}
$$

is a frequency-doubling factor.

The maximum energy emission occurs when $\mathrm{d} \phi / \mathrm{d} n=0$; according to Eq. (2.2), this corresponds to a population inversion $n_{\mathrm{t}}$ given by the transcendental equation

$$
\frac{1}{1-k}\left[1-\left(\frac{n_{\mathrm{t}}}{n_{\mathrm{i}}}\right)^{1-k}\right]=(1-\delta) p \frac{\alpha}{\alpha-k}\left[1-\left(\frac{n_{\mathrm{t}}}{n_{\mathrm{i}}}\right)^{\alpha-k}\right],
$$

where $p=-\ln T_{0}^{2} /\left(-\ln R+L-\ln T_{0}^{2}\right)$. It is worthwhile to mention that for a fixed value of SA initial transmission $T_{0}$ the parameter $p$ increases as the output mirror reflectivity $R$ increases. Alternatively, if $R$ is maintained constant, $p$ decreases as a SA crystal with higher $T_{0}$ is used.

The final population inversion, $n_{\mathrm{f}}$, is obtained by setting (2.2) equal to zero yielding the transcendental equation

$$
\begin{gathered}
\frac{1}{1-k}\left[1-\left(\frac{n_{\mathrm{f}}}{n_{\mathrm{i}}}\right)^{1-k}\right]+\frac{1}{k}[1-(1-\delta) p]\left[1-\left(\frac{n_{\mathrm{f}}}{n_{\mathrm{i}}}\right)^{-k}\right] \\
=\frac{1}{\alpha-k}(1-\delta) p\left[1-\left(\frac{n_{\mathrm{f}}}{n_{\mathrm{i}}}\right)^{\alpha-k}\right] .
\end{gathered}
$$

The peak power of second harmonic generation (SHG) is given by [7]:

$$
P_{\mathrm{p}}=\frac{h \nu A_{\mathrm{g}}}{2 \sigma \gamma t_{\mathrm{r}}}\left(2 \sigma n_{\mathrm{i}} l\right)^{2} u,
$$

where the normalized pulse peak power 


$$
u=\frac{1}{k}\left\{\frac{n_{\mathrm{t}}}{n_{\mathrm{i}}}-1+(1-\delta) p\left[1-\left(\frac{n_{\mathrm{t}}}{n_{\mathrm{i}}}\right)^{\alpha}\right]\right\}^{2}
$$

and the pulse energy of SHG is [7]:

$$
E_{\mathrm{p}}=\frac{A_{\mathrm{g}} h \nu n_{\mathrm{i}} l}{\gamma} e
$$

where

$$
e=1-\frac{n_{\mathrm{f}}}{n_{\mathrm{i}}}-\frac{1}{\alpha}(1-\delta) p\left[1-\left(\frac{n_{\mathrm{f}}}{n_{\mathrm{i}}}\right)^{\alpha}\right]+[1-(1-\delta) p] \ln \left(\frac{n_{\mathrm{f}}}{n_{\mathrm{i}}}\right) .
$$

The normalized pulse duration, $t_{\mathrm{p}}$ can be estimated as $t_{\mathrm{p}}=e / u$. However, as the real shape of the pulse was not taken into account, disagreement with the experimental value is likely to occur. To investigate the symmetry of the pulse shape a new parameter, $s_{\mathrm{p}}$, was defined by

$$
s_{\mathrm{p}}=\frac{E_{1}}{E_{\mathrm{p}}}
$$

where $E_{1}$ is the part of the pulse energy before the peak power is reached. The pulse symmetry parameter can be rewritten as follows:

$$
s_{\mathrm{p}}=\frac{e_{1}}{e}
$$

where [7]:

$$
\begin{aligned}
e_{1}= & 1-\left(1+\frac{1}{k}\right) \frac{n_{\mathrm{t}}}{n_{\mathrm{i}}}-\frac{1}{\alpha}(1-\delta) p\left[1-\left(1+\frac{\alpha}{k}\right)\left(\frac{n_{\mathrm{t}}}{n_{\mathrm{i}}}\right)^{\alpha}\right] \\
& +[1-(1-\delta) p]\left[\ln \left(\frac{n_{\mathrm{t}}}{n_{\mathrm{i}}}\right)+\frac{1}{k}\right] .
\end{aligned}
$$

Since immediately after the pulse emission the photon flux can be considered as negligible and the SA ground state population is very rapidly occupied compared to the build-up time for $n$, Eq. (2.1b) with $\phi=0$ can be integrated to obtain the evolution of the population density $n$ under CW pumping

$$
n(t)=W_{\mathrm{p}} \tau_{\mathrm{g}}-\left(W_{\mathrm{p}} \tau_{\mathrm{g}}-n_{\mathrm{f}}\right) \exp \left(-t / \tau_{\mathrm{g}}\right)
$$

When this population density reaches the value $n_{\mathrm{i}}$, a new laser pulse is generated. Thus Eq. (2.5), (2.13) and $n_{\mathrm{i}}=\left(-\ln R+L-\ln T_{0}^{2}\right) / 2 \sigma l$ (by setting Eq. (2.1a) equal to zero) enable the calculation of the repetition rate of the passively Q-switched pulses under CW pumping

$$
f=\frac{1}{\tau_{\mathrm{g}}} \ln \left(\frac{W_{\mathrm{p}} \tau_{\mathrm{g}}-n_{\mathrm{i}}}{W_{\mathrm{p}} \tau_{\mathrm{g}}-n_{\mathrm{f}}}\right) .
$$

\section{Numerical results}

Figures 3 and 4 present the variation of the relative inversion of population at the maximum energy emission $n_{\mathrm{t}} / n_{\mathrm{i}}$ and at the final inversion of population $n_{\mathrm{f}} / n_{\mathrm{i}}$, respectively, as a function of the parameter $p$. The ratio $n_{\mathrm{t}} / n_{\mathrm{i}}$ increases as $p$ decreases. At low values of $R$ or high $T_{0}$ a small fraction of the initial inversion 

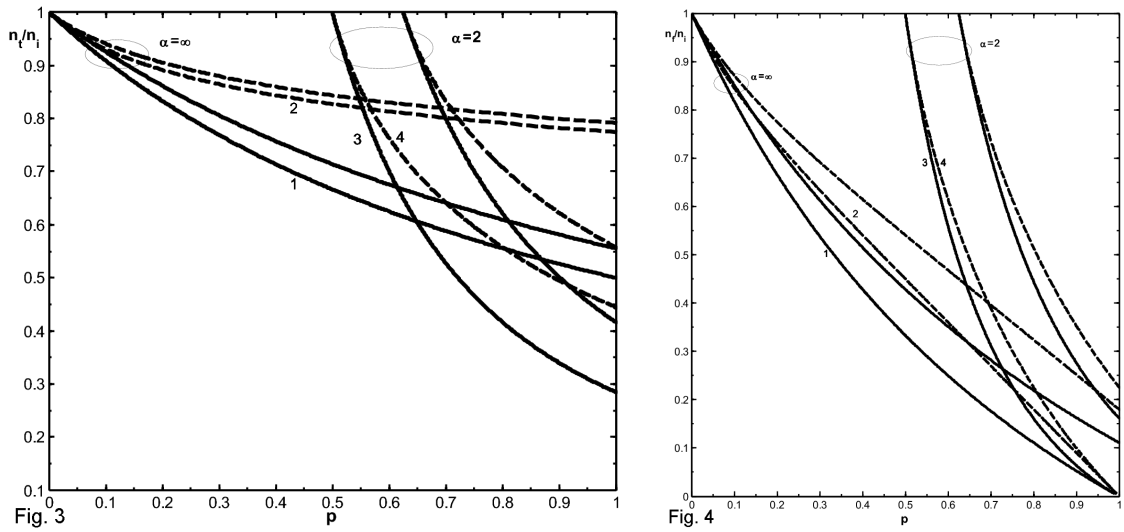

Fig. 3. The dependence of relative inversion of population on the normalized parameter $p$, defined in text, at maximum energy. Continuous lines correspond to $k=2$ and dashed lines to $k=10$. Curves $1-4$ correspond to $\delta=0$ and the other curves correspond to $\delta=0.2$.

Fig. 4. The dependence of relative inversion of population on the normalized parameter $p$, at the end of Q-switched pulse. Continuous lines correspond to $k=2$ and dashed lines to $k=10$. Curves $1-4$ correspond to $\delta=0$ and the other curves correspond to $\delta=0.2$.
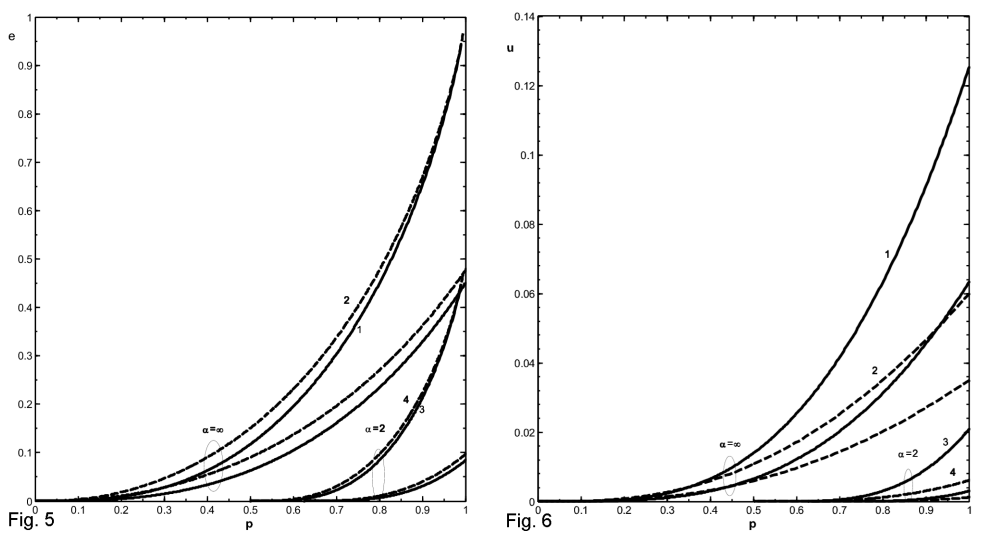

Fig. 5. Dependence of the normalized pulse energy $e$ on $p$ for various values of $\alpha, \delta$, and $k$. Continuous lines correspond to $k=2$ and dashed lines to $k=10$. Curves $1-4$ correspond to $\delta=0$ and the other curves correspond to $\delta=0.2$.

Fig. 6. Normalized pulse peak power $u$ as function of $p$ for various values of $\alpha, \delta$, and $k$. Continuous lines correspond to $k=2$ and dashed lines to $k=10$. Curves $1-4$ correspond to $\delta=0$ and the other curves correspond to $\delta=0.2$.

of population is used to build the laser pulse. Consequently, the final inversion of population $n_{\mathrm{f}} / n_{\mathrm{i}}$ is high and a low fraction of the initial inversion of population 

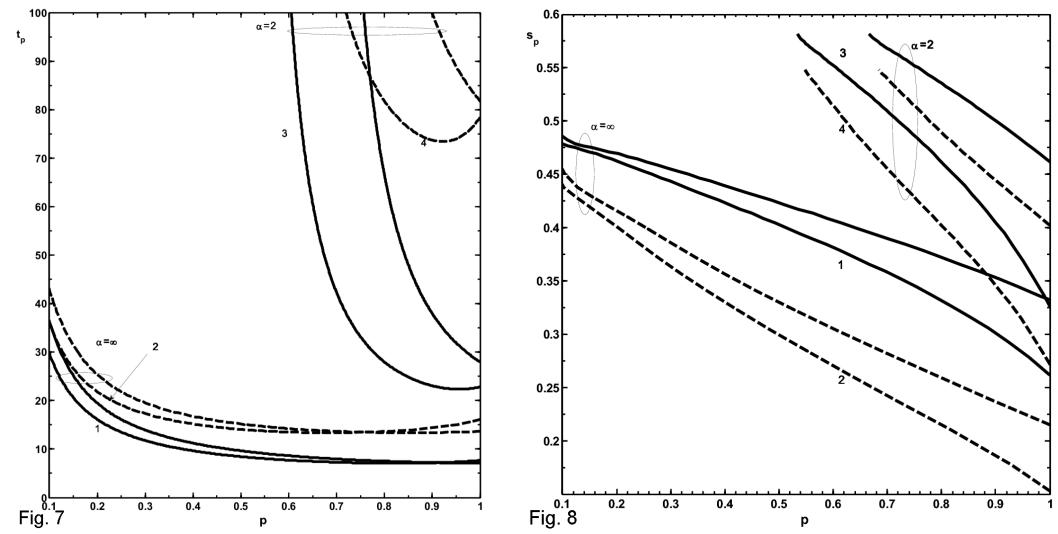

Fig. 7. Normalized pulse width $t_{\mathrm{pn}}$ as function of $p$ for various values of $\alpha, \delta$, and $k$. Continuous lines correspond to $k=2$ and dashed lines to $k=10$. Curves $1-4$ correspond to $\delta=0$ and the other curves correspond to $\delta=0.2$.

Fig. 8. Pulse symmetry $s_{\mathrm{p}}$ as function of $p$ for various values of $\alpha, \delta$, and $k$. Continuous lines correspond to $k=2$ and dashed lines to $k=10$. Curves $1-4$ correspond to $\delta=0$ and the other curves correspond to $\delta=0.2$.

contributes to the laser pulse. For all values of $p$ we can decrease $n_{\mathrm{t}} / n_{\mathrm{i}}$ and $n_{\mathrm{f}} / n_{\mathrm{i}}$ by increasing the parameter $\alpha$ or by decreasing the parameter $k$. The first condition can be achieved by choosing a resonator with an improved value of $A_{\mathrm{g}} / A_{\mathrm{s}}$, while the other one can be achieved by choosing a nonlinear optical crystal with small conversion efficiency.

The normalized pulse energy $e$ is presented in Fig. 5. For a fixed value of $R$, the pulse energy can be increased by decreasing the SA initial transmission $T_{0}$. However, as $T_{0}$ decreases the threshold inversion of population increases, which means a higher pumping rate is required and the laser will start to oscillate at lower repetition rates. Figure 6 shows the normalized pulse peak power $u$ as a function of $p$. It is clear that decreasing the SA initial transmission $T_{0}$ leads to obtain Q-switched pulses with higher peak power.

The normalized pulse width $t_{\mathrm{p}}$ and the pulse symmetry parameter $s_{\mathrm{p}}$ are presented in Figs. 7 and 8, respectively. In the low energy and low peak power regime the Q-switched laser delivers pulses characterized by long duration and high symmetry. Normally, pulses of higher harmonic peak power and higher energy with shorter pulse width are needed, which means poorer pulse symmetry.

\section{Conclusion}

We generate some design curves such as peak power, energy, pulse width, and a factor describing the pulse symmetry as functions of the variable $p$ for various values of $\alpha, \delta$, and $k$. With these curves and expressions, we demonstrate how these parameters could affect the design of a passively Q-switched intracavity frequency-doubled laser and predict the pulse characteristics. 
The peak power is expected to increase as the resonator length decreases. Inserting a focusing lens in the cavity could optimize the average output power and a polarized beam is expected with a plate positioned at the Brewster angle.

\section{Acknowledgments}

The authors would like to express their gratitude to the Director General of AECS Prof. I. Othman for his continuous encouragement, guidance and support.

\section{References}

[1] S. Zhao, L. Chen, G. Li, K. Yang, D. Li, L. Zhang, Opt. Quantum Electron. 36, 1227 (2004).

[2] T.T. Kajava, L.G. Alexander, Opt. Commun. 137, 93 (1997).

[3] K. Yang, S. Zhao, G. Li, J. Zou, J. Opt. Soc. Am. B 23, 671 (2006).

[4] G. Li, S. Zhao, K. Yang, J. Liu, Opt. Quantum Electron. 37, 635 (2005).

[5] G. Li, S. Zhao, H. Zhao, K. Yang, S. Ding, Opt. Commun. 234, 321 (2004).

[6] A. Brenier, C. Tu, Z. Zhu, J. Li, Appl. Phys. Lett. 90, 071103 (2007).

[7] J. Liu, D. Kim, IEEE J. Quantum Electron. 35, 1724 (1999).

[8] G. Xiao, M. Bass, IEEE J. Quantum Electron. 33, 41 (1997). 